\title{
A Wrong Argument in a Seminal Physics Paper
}

\author{
A. Leon \\ Dept. of Ciencias Naturales, I.E.S. Francisco Salinas, Salamanca, Spain (Retired) \\ Author's aail id: aleon.science@gmail.com
}

Available online at: www.isroset.org

Received: 09/Jan/2020, Accepted: 10/Feb/2020, Online: 28/Feb/2020

\begin{abstract}
This article examines a wrong argument in a seminal physics paper published 114 years ago: Einstein's paper on the electrodynamics of moving objects. Although the argument in question is not determinant for the main conclusions of the paper (thanks to the prevalence of Lorentz transformation also deduced in the same paper), it contains several basic and significant errors that have remained undetected to this day. Those errors are consequences of a misinterpretation of the Principle of Relativity, surprisingly the same misinterpretation one can find in some naïve critiques of special relativity. Considering the relevance of the article and of its author and the importance of the errors, there is no alternative but to make them public.
\end{abstract}

Keywords - Principle of relativity, special relativity, relativity of simultaneity, Einstein test for synchronous clocks.

\section{INTRODUCTION}

Errors are an invaluable source of experience and knowledge. But, obviously, errors should be detected and corrected. And errors in seminal papers must be noticed to avoid their dissemination and to facilitate the understanding of the birth, reception, and development of the new ideas usually introduced in seminal papers. In the case of the errors examined here, it is striking that they have never been noticed because, even not being conclusive for the main objective of the paper, they are really evident and important, and illustrate Einstein's wrong way of dealing with the Principle of Relativity and the relativity of simultaneity. It seems convenient to recall that from the title of the paper, from its introductory text [1, pp. 891-892], and from Einstein's own words 48 years after its publication (letter to Carl Seelig dated in 1953 [2, p. 104]), the main objective of Einstein's article was not to lay the foundation of special relativity as a new theory of spacetime, but to apply Lorentz transformation to Maxwell's equations of electromagnetism. This could have been the reason for which Einstein did not properly revised the $\$ 2$ of his paper (a short discussion on the relativity of lengths and times) that contains the wrong argument. In any case, much more important than the errors themselves is the fact that they have not been detected for more than 114 years, Einstein's paper being one of the most read (?) and cited works of modern physics, and the errors being so basic and evident (as the reader will immediately see). This anomalous circumstance should merit the attention and concern of contemporary physicists. And the errors should be noticed according to their importance, and to the influence of the paper and of its author.
In $\S 2$ of his paper, Einstein introduces equation (7) through the sentence "we find that (finden wir)" [1, p. 896], without giving details on where (7) comes from. It was by searching where (7) comes from that I discovered Einstein's error. And there is no doubt it comes from a misinterpretation of the Principle of Relativity. As Section II notes, such a misinterpretation is revealed for the first time in this article. Einstein's paper is introduced in Section III, and his argument on the relativity of lengths and times in Section IV. Then, in Section V, Einstein's errors are explained in detail. And Section VI summarizes them and some of their consequences.

\section{RELATED WORK}

In several footnotes of his Spanish translation of Einstein's 1905 articles, Antonio Ruiz de Elvira makes some critical comments on the obscurity of some Einstein arguments [3]. Apart from this criticism, and as far as I know, the present paper reveals for the first time a significant wrong argument in Einstein's paper on the electrodynamics of moving objects published in 1905.

\section{MATERIALS: A SEMINAL PHYSICS PAPER}

Einstein's paper "Zur Elektrodynamik bewegter Körper" [1] is divided into two parts, the Kinematical Part and the Electrodynamical Part. In the Kinematical Part, and after a short discussion on the relativity of lengths and times that includes the statements of the two principles of the special relativity, Einstein deduces, according to both principles, Lorentz transformation (LT from now on), as well as a formula for the composition of velocities. The Electrodynamical Part applies these results to Maxwell's 
equations of electromagnetism and to other electrodynamics questions. The erroneous argument examined here is in the $\$ 2$ of the Kinematical Part, entitled "On the Relativity of Lengths and Times". The English translation of the Einstein's texts quoted in this work is in the public domain [4] and has been contrasted with French [5] and Spanish [3] translations as well as with the original paper in German language [1].

\section{THE RELATIVITY OF SIMULTANEITY}

In $\S 1$ of his article, Einstein defines a criterion (EC hereafter) to test the synchronous state of two clocks placed at two points A and B [1, pp. 893-894] (equation number is mine):

«If at the point A of space there is a clock [...] If there is at the point $B$ of space another clock in all respects resembling the one at $\mathrm{A}[\ldots]$ we establish by definition that the "time" required by light to travel from $\mathrm{A}$ to $\mathrm{B}$ equals the "time" it requires to travel from B to A. Let a ray of light start at the "A-time" $t_{\mathrm{A}}$ from A towards B, let it at the "Btime" $t_{B}$ be reflected at $B$ in the direction of $A$, and arrive again at $A$ at the "A-time" $t_{A}^{\prime}$. In accordance with definition the two clocks synchronize if:

$$
\mathrm{t}_{\mathrm{B}}-\mathrm{t}_{\mathrm{A}}=\mathrm{t}_{\mathrm{A}}-\mathrm{t}_{\mathrm{B}}
$$

In accordance with the above Einstein's text, the time $t_{A B}$ light travels when going from $A$ to $B$ equals the time $t_{B A}$ it travels when going back from B to A. So, and according to the Second Principle of Relativity, the distance $\mathrm{d}_{\mathrm{AB}}$ light travels from $A$ to $B$ equals the distance $d_{B A}$ it travels when going back from $\mathrm{B}$ to $\mathrm{A}$. This is what the following corollary makes explicit and proves:

EC corollary: If $\mathrm{d}_{\mathrm{AB}} \neq \mathrm{d}_{\mathrm{BA}}$, then $\mathrm{EC}$ is not conclusive.

Proof.-Let $\mathrm{d}_{\mathrm{AB}} \neq \mathrm{d}_{\mathrm{BA}}$ and assume the clock at $\mathrm{A}$ is ahead of the clock at $B$ by a time $t^{*} \geq 0$ (a similar argument applies if the clock at B is ahead of the clock at A). According to the Second Principle of Relativity, we can write:

$$
\begin{gathered}
\mathrm{d}_{\mathrm{AB}} \neq \mathrm{d}_{\mathrm{BA}} \Leftrightarrow \mathrm{t}_{\mathrm{AB}} \neq \mathrm{t}_{\mathrm{BA}} \\
\mathrm{t}_{\mathrm{B}}=\mathrm{t}_{\mathrm{A}}+\mathrm{t}_{\mathrm{AB}}-\mathrm{t}^{*} ; \mathrm{t}^{\prime}{ }_{\mathrm{A}}=\mathrm{t}_{\mathrm{A}}+\mathrm{t}_{\mathrm{AB}}+\mathrm{t}_{\mathrm{BA}} \\
\mathrm{t}_{\mathrm{B}}-\mathrm{t}_{\mathrm{A}}=\mathrm{t}_{\mathrm{AB}}-\mathrm{t}^{*} ; \mathrm{t}^{\prime}{ }_{\mathrm{A}}-\mathrm{t}_{\mathrm{B}}=\mathrm{t}_{\mathrm{BA}}+\mathrm{t}^{*} \\
\mathrm{t}_{\mathrm{B}}-\mathrm{t}_{\mathrm{A}}=\mathrm{t}^{\prime}{ }_{\mathrm{A}}-\mathrm{t}_{\mathrm{B}} \Leftrightarrow \mathrm{t}_{\mathrm{AB}}-\mathrm{t}^{*}=\mathrm{t}_{\mathrm{BA}}+\mathrm{t}^{*} \\
\mathrm{t}_{\mathrm{AB}}-\mathrm{t}^{*}=\mathrm{t}_{\mathrm{BA}}+\mathrm{t}^{*} \Leftrightarrow \mathrm{t}_{\mathrm{AB}}-\mathrm{t}_{\mathrm{BA}}=2 \mathrm{t}^{*}
\end{gathered}
$$

Therefore, if $\mathrm{d}_{\mathrm{AB}} \neq \mathrm{d}_{\mathrm{BA}}$ and $\mathrm{t}^{*}=0$, both clocks are synchronous, but EC gives them as asynchronous because of (2) and (4): $t_{B}-t_{A}=t_{A B} \neq t_{B A}=t_{A}^{\prime}-t_{B}$. On the other hand, if $\mathrm{d}_{\mathrm{AB}} \neq \mathrm{d}_{\mathrm{BA}}$ and $\mathrm{t}^{*}>0$, both clocks are asynchronous, but $\mathrm{EC}$ gives them as synchronous if $\mathrm{t}_{\mathrm{AB}}$ $\mathrm{t}_{\mathrm{BA}}=2 \mathrm{t}^{*}(5)-(6)$. In consequence, if $\mathrm{d}_{\mathrm{AB}} \neq \mathrm{d}_{\mathrm{BA}}, \mathrm{EC}$ is not conclusive.

In $\$ 2$ of his paper, and before deducing LT, Einstein tries to prove the synchronous state of two clocks is not the same for all observers [1, pp. 896-897] (emphasis and paragraphs and equation numbering is mine):
«[P1]. Let there be given a stationary rigid rod; and let its length be $l$ as measured by a measuring rod which is also stationary. We now imagine the axis of the rod lying along the $\mathrm{X}$ axis of the stationary system of coordinates, and that a uniform motion of parallel translation with velocity $\mathrm{V}$ along the $\mathrm{X}$ axis in the direction of increasing $\mathrm{x}$ is then imparted to the rod. [...]

[P2]. We imagine further that at the two ends A and B of the rod, clocks are placed which synchronize with the clocks of the stationary system, that is to say that their indications correspond at any instant to the "time of the stationary system" at the places where they happen to be. These clocks are therefore "synchronous in the stationary system."

[P3]. We imagine further that with each clock there is a moving observer, and that these observers apply to both clocks the criterion established in $\$ 1$ for the synchronization of two clocks. Let a ray of light depart from $A$ at the time $^{1} t_{A}$, let it be reflected at $B$ at the time $t_{B}$, and reach $A$ again at the time $t_{A}^{\prime}$. Taking into consideration the principle of the constancy of the velocity of light [V], we find that:

$$
t_{B}-t_{A}=r_{A B} /(V-v) \text { and } t^{\prime}{ }_{A}-t_{B}=r_{A B} /(V+v)
$$

where $\mathrm{r}_{\mathrm{AB}}$ denotes the length of the moving rod measured in the stationary system. Observers moving with the moving rod would thus find that the two clocks were not synchronous, while observers in the stationary system would declare the clocks to be synchronous.»

\section{DISCUSSION: EINSTEIN WRONG ARGUMENT}

According to Einstein's definition of EC, and taking into account that "with each clock [placed at the end of the rod] there is a moving observer" and that "these [moving] observers apply [EC] to both clocks" [P3], both clocks must be at rest relative to the moving observers and in their immediate proximity, so that they must also move with the same uniform velocity $\mathrm{v}$ relative to the stationary system as the moving observers and the moving rod while the moving observers apply EC to them. This (obviousness) will be confirmed next by (8)-(-17), and then by Einstein's (7). For this reason, the next discussion makes use of the following nomenclature (in addition to Einstein's): the stationary observers and their inertial rest frame will be referred to as s-observers and Fs respectively; the moving observers, which carry out all EC measurements, will be referred to as m-observers; $\mathrm{Ac}$ and $\mathrm{Bc}$ will denote the moving clocks placed at the respective ends $A$ and $B$ of the moving rod; the rod, the clocks Ac, Bc and m-observers move with the same uniform velocity $\mathrm{v}$ relative to Fs in the direction of the increasing $\mathrm{x}$ of the $\mathrm{X}$ axis of Fs, which is also the direction from $\mathrm{A}$ to $\mathrm{B}$; the inertial rest frame of the rod, the clocks Ac, Bc and m-observers will be denoted by

\footnotetext{
1 Time" here denotes "time of the stationary system" and also "position of hands of the moving clock situated at the place under discussion." [Einstein's original footnote]
} 
Fm; $\mathrm{d}_{\mathrm{AB}}, \mathrm{d}_{\mathrm{BA}}$ will respectively denote the distances light travels from $\mathrm{Ac}$ to $\mathrm{Bc}$ and then from $\mathrm{Bc}$ to $\mathrm{Ac}$; and $\mathrm{c}$ denotes Einstein's speed of light V.

If m-observers refer their EC measurements to their rest frame Fm, they will find that $d_{A B}=d_{B A}=l$, from which they cannot get Einstein's (7), which comes from:

$$
\begin{gathered}
\mathrm{t}_{\mathrm{A}}<\mathrm{t}_{\mathrm{B}}<\mathrm{t}^{\prime}{ }_{\mathrm{A}} \\
\mathrm{d}_{\mathrm{AB}}=\mathrm{r}_{\mathrm{AB}}+\mathrm{v}\left(\mathrm{t}_{\mathrm{B}}-\mathrm{t}_{\mathrm{A}}\right) \\
\mathrm{d}_{\mathrm{BA}}=\mathrm{r}_{\mathrm{AB}}-\mathrm{v}\left(\mathrm{t}_{\mathrm{A}}-\mathrm{t}_{\mathrm{B}}\right) \\
\mathrm{t}_{\mathrm{B}}-\mathrm{t}_{\mathrm{A}}=\mathrm{d}_{\mathrm{AB}} / \mathrm{c}=\left(\mathrm{r}_{\mathrm{AB}}+\mathrm{v}\left(\mathrm{t}_{\mathrm{B}}-\mathrm{t}_{\mathrm{A}}\right)\right) / \mathrm{c} \\
\mathrm{t}^{\prime} \mathrm{A}_{\mathrm{A}}-\mathrm{t}_{\mathrm{B}}=\mathrm{d}_{\mathrm{BA}} / \mathrm{c}=\left(\mathrm{r}_{\mathrm{AB}}-\mathrm{v}\left(\mathrm{t}^{\prime}{ }_{\mathrm{A}}-\mathrm{t}_{\mathrm{B}}\right)\right) / \mathrm{c} \\
\mathrm{c}\left(\mathrm{t}_{\mathrm{B}}-\mathrm{t}_{\mathrm{A}}\right)=\mathrm{r}_{\mathrm{AB}}+\mathrm{v}\left(\mathrm{t}_{\mathrm{B}}-\mathrm{t}_{\mathrm{A}}\right) \\
\mathrm{c}\left(\mathrm{t}^{\prime}{ }_{\mathrm{A}}-\mathrm{t}_{\mathrm{B}}\right)=\mathrm{r}_{\mathrm{AB}}-\mathrm{v}\left(\mathrm{t}_{\mathrm{A}}{ }-\mathrm{t}_{\mathrm{B}}\right) \\
\left(\mathrm{t}_{\mathrm{B}}-\mathrm{t}_{\mathrm{A}}\right)(\mathrm{c}-\mathrm{v})=\mathrm{r}_{\mathrm{AB}} \\
\left(\mathrm{t}_{\mathrm{A}}-\mathrm{t}_{\mathrm{B}}\right)(\mathrm{c}+\mathrm{v})=\mathrm{r}_{\mathrm{AB}} \\
\mathrm{t}_{\mathrm{B}}-\mathrm{t}_{\mathrm{A}}=\mathrm{r}_{\mathrm{AB}} /(\mathrm{c}-\mathrm{v}) \text { and } \mathrm{t}_{\mathrm{A}}-\mathrm{t}_{\mathrm{B}}=\mathrm{r}_{\mathrm{AB}} /(\mathrm{c}+\mathrm{v})
\end{gathered}
$$

Therefore, and unusual as it may be, m-observers do not refer their EC measurements to their rest frame Fm. The only possible alternative is that m-observers refer their EC measurements to Fs, either as a moving frame or as a stationary frame. In fact, both alternatives, and only them, lead to Einstein's (7) through (8)-(17). But these alternatives imply that, according to (8)-(10), light travels a distance $d_{A B}$ (9) when going from $A c$ to $B c$ greater than the distance $d_{B A}$ (10) it travels when going back from $\mathrm{Bc}$ to Ac. In these conditions, EC does not work because of EC corollary. So, Einstein cannot claim that, according to the EC carried out by m-observers, Ac and Bc are not synchronous for m-observers because of (7), given that (7) comes from (8)-(17), which implies $d_{A B} \neq d_{B A}$ (8)-(10), and EC is not conclusive if $d_{A B} \neq$ $\mathrm{d}_{\mathrm{BA}}$ (EC corollary). Alternatively, starting from (17), going back to (14)-(13), and taking into account that $t_{A}, t_{B}$ and $t^{\prime}{ }_{A}$ are Fs times of Fs' synchronous clocks (see Einstein's footnote), $c\left(t_{B}-t_{A}\right)$ and $c\left(t_{A}^{\prime}-t_{B}\right)$ are respectively the distances $r_{A B}+v\left(t_{B}-t_{A}\right)$ and $r_{A B}-v\left(t_{A}-t_{B}\right)$ that light travels in Fs when going from the moving $\mathrm{Ac}$ to the moving $\mathrm{Bc}$, and then when going back from the moving $\mathrm{Bc}$ to moving $\mathrm{Ac}$, which confirms that m-observers refer their EC measurements to Fs.

In consequence, Fs would be a sort of universal frame of reference to which not only s-observers but also m-observers refer their corresponding measurements. So, and being all measurements referred to $\mathrm{Fs}$, it must be concluded that, according to Einstein's argument [P1]-[P3], the clocks Ac and $\mathrm{Bc}$ are (s-observers), and are not (m-observers), synchronous in the same frame Fs. And the same would apply to any other measurement carried out relative to Fs by s-observers and by m-observers. These contradictory results are the consequences of a misinterpretation of the Principle of Relativity. Indeed, the Principle of Relativity of special relativity requires that each observer refers all his measurements to his rest frame, otherwise contradictory results, as the above one, are inevitable.

In $§ 3$ Einstein deduces LT, from which the relativity of simultaneity immediately follows, making it unnecessary the above erroneous argument [P1]-[P3]. According to LT, if two clocks separated by a proper distance $\mathrm{x}$ are synchronous in their proper inertial frame, from another inertial frame relative to which the clocks move at a uniform velocity $\mathrm{v}$ in the direction in which they are separated by the proper distance $\mathrm{x}$, the chasing clock is observed ahead of the other by a time $\mathrm{xv} / \mathrm{c}^{2}$. Maybe Einstein was not aware of this genuine relativity of simultaneity linked to LT because, apart from the wrong [P1]-[P3], he only comments the loss of synchronism between two clocks as a consequence of time dilation, also linked to LT [1, p. 904].

\section{CONCLUSIONS}

The above discussion on Einstein's argument [P1]-[P3] reveals it contains several significant errors based on a misinterpretation of the Principle of Relativity: m-observers do not refer their measurements on EC to their own rest frame but to the moving reference frame of s-observers, which is a rude relativist error we can found in many naïve interpretations of the special theory of relativity. In consequence, Einstein's [P1]-[P3] does not prove the relativity of simultaneity but a contradiction: the clocks Ac and $\mathrm{Bc}$ are and are not synchronous in the same reference frame Fs. The relativity of simultaneity is a direct consequence of Lorentz transformation, also deduced in Einstein's paper.

It seems reasonable to assume that Einstein's paper has been read by thousands of scientifically educated readers all around the world, despite of which the erroneous nature of the argument [P1]-[P3] has remained unnoticed for more than 114 years. This points to a deficit of criticism and an excess of reverence for certain authors and theories in contemporary science.

\section{REFERENCES}

[1] A. Einstein, "Zur Elektrodynamik bewegter Körper", Annalen der Physik, vol. 17, pp. 891-921, 1905.

[2] M. Born, "Physics in my generation. A selection of papers", Springer-Verlag, New York, 1969.

[3] A. R. Elvira, "Cien años de relatividad. Los artículos clave de 1905 y 1906," Nivola, Madrid, 2003.

[4] H. A. Lorentz, A. Einstein, H. Minkowski, H. Weyl and A. Sommerfeld, "The Principle of Relativity", Methuen and Company, London, 1923.

[5] A. Einstein, "De l'eléctrodynamique des corpss en mouvement", Les classiques des sciences sociales, Université de Québec, 2012.

\section{AUTHORS PROFILE}

Graduated in Geology, two years of specialization in Crystallography (including Solid-State Physics), regular studies in Mathematical Sciences and a Doctorate Program in Philosophy and History of Science. He has worked as a professor of

Natural Sciences, Mathematics and Computer Science at high schools. He is now retired from his teaching activity. For more than forty years he has been an independent researcher in the field of the formal foundations of science, particularly the foundation of mathematics and physics. Some of his works are published in peer reviewed journals and, as an invited author, in a book chapter (on physics and the Axiom of Infinity). 